\title{
JunD protects against chronic kidney disease by regulating paracrine mitogens
}

\author{
Evangéline Pillebout, ${ }^{1}$ Jonathan B. Weitzman, ${ }^{2}$ Martine Burtin, ${ }^{1}$ Carla Martino, ${ }^{1}$ \\ Pierre Federici, ${ }^{1}$ Moshe Yaniv, ${ }^{2}$ Gérard Friedlander, ${ }^{1}$ and Fabiola Terzi ${ }^{1}$ \\ ${ }^{1}$ Institut National de la Santé et de la Recherche Médicale U426, Department of Physiology, Faculté de Médecine Xavier Bichat, \\ Paris, France \\ ${ }^{2}$ Centre National de La Recherche Scientifique URA1644, Unit of Gene Expression \& Disease, Department of Developmental \\ Biology, Institut Pasteur, Paris, France
}

\begin{abstract}
The AP- 1 transcription factor, composed of Jun and Fos proteins, plays a crucial role in the fine tuning of cell proliferation. We showed previously that AP-1 complexes are activated during the proliferative response that parallels the development of renal lesions after nephron reduction, but little is known about the specific role of individual Jun/Fos components in the deterioration process. Here we used JunD knockout $\left(\right.$ JunD $\left.^{-/}\right)$mice and an experimental model of chronic renal injury $(75 \%$ nephron reduction) to explore the role of JunD. Nephron reduction resulted in an initial compensatory growth phase that did not require JunD. JunD, however, was essential to inhibit a second wave of cell proliferation and to halt the development of severe glomerular sclerosis, tubular dilation, and interstitial fibrosis. We show that the effects of $j u n D$ inactivation are not cell autonomous and involve upregulation of the paracrine mitogen, TGF- $\alpha$. Expression of a transgene (REM) encoding a dominant negative isoform of the EGFR, the receptor for TGF- $\alpha$, prevented the second wave of cell proliferation and the development of renal lesions in bitransgenic JunD $D^{-/} / R E M$ mice. We propose that JunD is part of a regulatory network that controls proliferation to prevent pathological progression in chronic renal diseases.
\end{abstract}

J. Clin. Invest. 112:843-852 (2003). doi:10.1172/JCI200317647.

\begin{abstract}
Introduction
Reduction of renal mass triggers molecular and cellular events promoting compensatory growth of remaining nephrons. In some cases, this compensatory process becomes pathological with the development of renal lesions and end-stage renal failure (1). Although the pathophysiology of compensation and progression is complex, deregulated proliferation of glomerular, tubular, and interstitial cells may promote the development of progressive glomerular sclerosis, tubular cysts, and interstitial fibrosis (2-5). Indeed, the reduction of renal cell proliferation, using pharmacological growth factor inhibitors (6), neutralizing Ab's (7), antisense oligonucleotides (8), or transgenic strategies (9), halts the progression of renal lesions in some experimental models of renal injury. The molecular mechanisms and the mediators underlying renal hyperplasia after nephron reduction remain largely unexplained.
\end{abstract}

Received for publication December 17, 2002, and accepted in revised form July 15, 2003.

Address correspondence to: Fabiola Terzi, Institut National de la Santé et de la Recherche Médicale U426, Faculté de Médecine, Xavier Bichat, BP416, 16, Rue Henri Huchard, 75870 Paris,

Cedex 18, France. Phone: 33-144856270; Fax: 33-142281564;

E-mail: terzi@bichat.inserm.fr.

Conflict of interest: The authors have declared that no conflict of interest exists.

Nonstandard abbreviations used: proliferating cell nuclear antigen (PCNA); periodic acid Schiff (PAS); lotus tetragonolobus lectin (LTL).
A complex balance of positive and negative secreted factors and cell-cell and cell-matrix interactions modulates the proliferation of renal cells under physiological and pathological conditions. These factors stimulate signaling pathways that converge to activate transcription factors and induce cell-cycle control genes. The AP-1 transcription factor acts downstream of mitogen-activated pathways and is often associated with cell proliferation (10). AP-1 is composed of Jun proteins (JunB, cJun, and JunD) that can form homodimers or heterodimers with Fos/ATF partners (10). Studies of AP-1 function in vitro have demonstrated distinct roles for each Jun protein and suggested that different dimer combinations determine the nature of AP-1-mediated cellular responses. JunD is broadly expressed, and its regulation differs markedly from $c-j u n$ and junB, both of which behave as immediate early genes upon mitogenic stimulation $(11,12)$. Overexpression of JunD suppresses fibroblast proliferation and antagonizes Ras-induced transformation, suggesting that JunD is a negative regulator of cell growth (13). In contrast, c-jun induces cell proliferation and transformation (13). Hence, differential regulation of the Jun proteins may coordinate distinct responses to extracellular mitogenic stimuli.

The role of AP- 1 in the evolution of renal lesions after injury is complex. In acute ischemic or toxic tubular injury, overexpression of c-Fos protein may contribute to the regeneration of damaged tubules by favoring cell proliferation (14). In contrast, in chronic renal disease activation of AP-1 has been associated with the deterioration 
process. AP-1 DNA-binding activity is increased during the development of glomerular and/or tubulo-interstitial lesions in several chronic pathological conditions, such as diabetes, hypertension, polycystic kidney disease, and glomerulonephrites (15-18). Subtotal nephrectomy induces the expression of $c$-fos and $c$-jun genes, cell proliferation, and renal lesions (3). Interfering with the development of renal lesions (either by dietary or by pharmacological modulations) decreases both AP-1 activity and cell proliferation in several experimental models of chronic renal injury $(5,18-20)$. Taken together, these results suggest that AP- 1 could be a major determinant in the development of renal lesions.

Genetically modified mice provide powerful tools to explore the functions of specific AP- 1 complexes in vivo (21). The use of these models in physiopathological studies is restricted, however. Indeed, disruption of $c-j u n$ or junB genes resulted in embryonic lethality, and fos knockout mice presented distinct phenotypes, that is, defects in bone development, growth, spermatogenesis, and animal behavior (21). In contrast, mice lacking junD are viable and display a mild phenotype with spermatogenesis defects (22). Analysis of $J u n D^{-/}$fibroblasts confirmed that JunD acts as a negative regulator of Ras signaling and cell proliferation and showed that the absence of JunD renders cells and mice susceptible to stress-induced apoptosis (23). The role of JunD in regulating cell proliferation in vivo is poorly defined.

Here, we have used JunD knockout mice to examine the role of JunD/AP-1 in the development of renal disease following nephron reduction. We show that the initial compensatory growth phase did not require JunD. JunD was essential to inhibit a second wave of cell proliferation, however, and to prevent the development of renal lesions. Moreover, we demonstrate that the effects of junD inactivation involve upregulation of the paracrine mitogen, TGF- $\alpha$.

\section{Methods}

Animals. JunD $D^{-/}$mice, in which the junD gene was replaced by a bacterial NLS- $\beta$-gal cassette, and REM mice, expressing a truncated EGFR under the control of kidney-specific type $1 \gamma$-glutamyl transpeptidase promoter, were previously described $(9,22)$. Homozygous $J u n D^{-/}$ mice were bred with homozygous REM mice to obtain double-homozygous transgenic JunD $D^{-/} / R E M$ mice. Mice are from mixed 129SV $\times$ C57Bl6 genetic backgrounds. All the experiments were performed on animals from the same litters. Animals were fed ad libitum and housed at constant ambient temperature in a 12hour light cycle. Animal procedures were conducted in accordance with French government policies (Services Vétérinaires de la Santé et de la Production Animale, Ministère de l'Agriculture).

Experimental protocol. Adult (3-4 months) $J u n D^{+/+}$and $J u n D^{-/-}$littermates were subjected to subtotal nephrectomy as described previously (24). Briefly, the right kidney was removed, and the two poles of the left kidney were excised to reach $75 \%$ reduction of total renal mass.
After surgery, mice were fed a defined diet containing $30 \%$ casein and $0.5 \%$ sodium. Mice were sacrificed at 7 , 14,30 , and 60 days after surgery. At each time point, four to six $J u n D^{+/+}$and $J u n D^{-/-}$mice were studied. In addition, bitransgenic $J u n D^{-/-} / R E M$ mice of the same age were subjected to subtotal nephrectomy $(n=10)$ and sacrificed 60 days after surgery. Six nonoperated mice of each group were used as controls.

At day 30, blood pressure was recorded in five awake mice of each experimental group for 3 consecutive days before sacrifice, using tail-cuff plethysmography and PowerLab/4SP software (AD Instruments, Paris, France). Urine samples were collected from the same animals using Marty Technologie (Paris, France) metabolic cages for the 24 hours before sacrifice.

Renal function. Urinary creatinine and protein concentrations were measured using an Olympus multiparametric autoanalyzer (Instrumentation Laboratory, Paris, France).

Renal morphology. Kidneys were fixed in $4 \%$ formaldehyde, paraffin embedded, and $4-\mu \mathrm{m}$ sections were stained with periodic acid Schiff (PAS), Masson's trichrome, $\mathrm{H} \& \mathrm{E}$, or picro-sirius red. A pathologist, blinded to the nature of the group, examined and evaluated all the sections.

Glomerular and tubular hypertrophy were measured on PAS-stained sections, using a Nikon digital camera $\mathrm{Dx} / \mathrm{m} / 1200$ and Lucia software (Laboratory Imaging Ltd., Prague, Czech Republic). Twenty randomly selected microscopic fields of the cortex were studied $(\times 200)$. At least 20 nonsclerotic glomeruli were analyzed for each animal. Tubular morphometry was performed on proximal tubules. Cross-sectional profiles were analyzed, tubules located near the scarred area, and tubules containing casts or showing evident dilation were excluded. The area of external profile and the area of the lumen were measured, and epithelial surface was calculated as the difference between these two areas. At least 35 tubular cross sections were analyzed for each animal. Tubular cell hyperplasia was evaluated on the same tubular cross sections by counting the number of nuclei. Tubular cell hypertrophy was calculated as the ratio between epithelial surface and the number of nuclei for tubular section.

The degree of renal lesions was quantified using an established semiquantitative score methodology (25) with minor modifications. Twenty randomly selected microscopic fields were scored. Glomerular lesions were evaluated on PAS-stained sections and graded from 0 to $2+$, according to the extent of mesangial cell proliferation, hyalinosis, and sclerosis. Tubular lesions were evaluated on PAS-stained sections and graded from 0 to $3+$, according to the severity of tubular dilations. The degree of interstitial fibrosis was determined on picro-sirius red-stained sections, using a $0-2+$ injury scale; 0,1 , and 2 correspond to $0 \%, 1-25 \%$, and $25-50 \%$ of the microscopic field. The degree of interstitial mononuclear cell infiltration was determined on H\&E-stained sections, using a $0-2+$ injury scale; 0,1 , and 2 correspond to $0 \%$, $1-10 \%$, and $10-20 \%$ of the microscopic field. 
Immunobistochemistry. Proliferating cell nuclear antigen (PCNA) immunostaining was performed as described previously (26). For $\beta$-gal detection, $4-\mu \mathrm{m}$ sections were incubated with a mouse anti- $\beta$-gal Ab (Sigma-Aldrich, St. Quentin-Fallavier, France) diluted 1:100, followed by a biotinylated sheep $\mathrm{Ab}$ (Amersham Pharmacia Biotech, Les Ulis, France), and 3-3'-diamino-benzidine-tetrahydrochloride (DAB). The number of PCNA and $\beta$-gallabeled tubular nuclei were determined in ten randomly selected fields of the cortex $(\times 200)$ and corrected for the number of tubular sections. Colocalization of PCNAand $\beta$-gal-labeled tubular nuclei was assessed in 20 randomly selected fields of serial sections.

For Tamm-Horsfall colocalization experiments, 4- $\mu \mathrm{m}$ sections were incubated with a sheep anti-human Tamm-Horsfall glycoprotein Ab (Valbiotech, Paris, France) diluted 1:50 and with either a mouse antiPCNA Ab diluted $1: 50$ or a mouse anti- $\beta$-gal Ab diluted 1:50. PCNA and $\beta$-gal was then detected as described above. Tamm-Horsfall protein was detected using a rabbit anti-goat Ab (AbCys Biology, Paris, France) conjugated with alkaline phosphatase diluted 1:150, followed by Vector blue alkaline phosphatase substrate (AbCys Biology). For lotus tetragonolobus lectin (LTL) colocalization experiments, $4-\mu \mathrm{m}$ sections were incubated with biotinylated LTL (AbCys Biology) diluted 1:50 and either with a mouse anti-PCNA Ab diluted $1: 50$ or a mouse anti- $\beta$-gal Ab diluted 1:50. PCNA and $\beta$-gal was then detected as described above. LTL was detected with a StreptABComplex/AP (DAKO, Trappes, France) followed by Vector blue alkaline phosphatase substrate. All sections were then counterstained by Vector methyl green (AbCys Biology).

For TGF- $\alpha$ and EGF detection, sections were incubated with goat anti-TGF- $\alpha$ Ab (Santa Cruz Biotechnology Inc., Santa Cruz, California, USA) diluted 1:200 or rabbit anti-EGF (Sigma-Aldrich) diluted 1:500, followed by biotinylated donkey secondary Ab's (Santa Cruz Biotechnology Inc.), the avidin/biotin/peroxidase system (LSAB2 kit; DAKO), and DAB. The specificity of TGF- $\alpha$ staining was confirmed using a fivefold excess of blocking peptide that eliminated all staining. The mean density of EGF and TGF- $\alpha$ staining was quantified with a Nikon digital camera $\mathrm{Dx} / \mathrm{m} / 1200$ and Lucia software in ten selected microscopic fields of the cortex $(\times 200)$.

Western blot analysis. Immunoblotting was performed as described previously (26) using a rabbit polyclonal anti-JunD Ab (13) diluted 1:1,000 or a goat polyclonal anti-TGF- $\alpha$ Ab (Santa Cruz Biotechnology Inc.) diluted 1:100. We used donkey horseradish peroxidase-linked secondary Ab's and enhanced chemiluminescence (Amersham Pharmacia Biotech). A mouse monoclonal anti- $\alpha$-tubulin Ab (Sigma-Aldrich) was used as a control. Protein extracts from kidneys of $J u n D^{-/-}$and TGF- $\alpha^{-/-}$mice (kindly provided by D.C. Lee, University of North Carolina, Chapel Hill, North Carolina, USA) were used to confirm Ab specificity.

Ribonuclease protection assay. The mRNA levels were quantified with a RNAse protection assay as described previously (27). EGF and TGF- $\alpha$ probes were generated by reverse transcriptase and PCR using these oligonucleotides: EGF primer, 5'-GACACATGCATTTTGATG and 5 '-TCCAGTAGATTCTCCG; TGF- $\alpha$ primers, 5 '-GCCCAGATTCCCACACTCAG and 5'-CACGGCACCACTCACAGTG. Cyclin D1, cyclin D2, cyclin D3, and control GAPDH cRNA probes were kindly provided by B. Lardeux (Institut National de la Santé et de la Recherche Médicale U327, Paris, France).

Data analysis and statistics. Data are expressed as means plus or minus SEM. Differences between the experimental groups were evaluated using ANOVA, followed, when significant, by the Tukey-Kramer test or the MannWhitney test when only two groups were compared.

Table 1

Renal morphometry at sacrifice

\begin{tabular}{|c|c|c|c|c|c|}
\hline Time after surgery & $\begin{array}{l}\mathrm{KW} / \mathrm{BW} \\
(\%)\end{array}$ & $\begin{array}{l}\text { Glomerular surface } \\
\left(\mu \mathrm{m}^{2}\right)\end{array}$ & $\begin{array}{c}\text { Tubular surface } \\
\left(\mu \mathrm{m}^{2}\right)\end{array}$ & $\begin{array}{l}\text { No. nuclei/tubular } \\
\text { section }\end{array}$ & $\begin{array}{l}\text { Cell size } \\
\left(\mu \mathrm{m}^{2}\right)\end{array}$ \\
\hline Day 0 & $0.46 \pm 0.02$ & $2,065 \pm 84$ & $616 \pm 28$ & $3.7 \pm 0.1$ & $165 \pm 9$ \\
\hline \multicolumn{6}{|l|}{ Day 7} \\
\hline JunD $D^{+/+}$ & $0.55 \pm 0.06$ & $2,424 \pm 25$ & $1,390 \pm 58^{c}$ & $4.9 \pm 0.3^{c}$ & $288 \pm 22^{C}$ \\
\hline JunD $D^{-/-}$ & $0.52 \pm 0.05$ & $2,490 \pm 95$ & $1,154 \pm 17 c$ & $4.7 \pm 0.3^{\mathrm{A}}$ & $245 \pm 15^{C}$ \\
\hline \multicolumn{6}{|l|}{ Day 14} \\
\hline JunD $D^{+/+}$ & $0.52 \pm 0.10$ & $2,661 \pm 39^{B}$ & $1,150 \pm 77 c$ & $4.9 \pm 0.1^{\mathrm{B}}$ & $237 \pm 15^{B}$ \\
\hline JunD $D^{-/-}$ & $0.55 \pm 0.04$ & $2,728 \pm 55^{\mathrm{B}}$ & $1,113 \pm 43 c$ & $5.1 \pm 0.1^{c}$ & $220 \pm 8^{c}$ \\
\hline \multicolumn{6}{|l|}{ Day 30} \\
\hline JunD $D^{+/+}$ & $0.56 \pm 0.08$ & $2,930 \pm 319 c$ & $1,363 \pm 76^{c}$ & $5.2 \pm 0.2^{\mathrm{C}}$ & $264 \pm 18^{c}$ \\
\hline JunD $D^{-/-}$ & $0.59 \pm 0.09$ & $2,809 \pm 88^{C}$ & $1,342 \pm 51^{c}$ & $4.8 \pm 0.2^{\mathrm{B}}$ & $279 \pm 13^{C}$ \\
\hline \multicolumn{6}{|l|}{ Day 60} \\
\hline JunD $D^{+/+}$ & $0.59 \pm 0.17$ & $2,684 \pm 155^{\mathrm{B}}$ & $1,741 \pm 46^{C}$ & $4.7 \pm 0.1^{\mathrm{A}}$ & $373 \pm 15^{c}$ \\
\hline JunD $D^{-/-}$ & $0.79 \pm 0.11$ & $3,458 \pm 59^{C, D}$ & $2,577 \pm 57 \mathrm{C,D}$ & $9.2 \pm 0.6^{\mathrm{C}, \mathrm{D}}$ & $284 \pm 15^{C, L}$ \\
\hline
\end{tabular}

Glomerular and tubular surfaces were measured in 20 randomly selected microscopic fields of the cortex in control kidneys (Day 0$)$ and in $J u n D^{+/+}$and $J u n D^{-/-}$ remnant kidneys $7,14,30$, and 60 days after nephron reduction. The number of tubular nuclei was counted on the same tubular sections. Data are mean \pm SEM; $n=4-6$ for each time point. ${ }^{A} P<0.05$, ${ }^{\mathrm{B}} P<0.01,{ }^{C} P<0.001$, remnant versus control kidneys; ${ }^{\mathrm{D}} P<0.001$, JunD ${ }^{-/-}$versus $J u n D^{+/+}$by $\mathrm{ANOVA}$, followed by Tukey-Kramer test. KW, kidney weight; BW, body weight. 

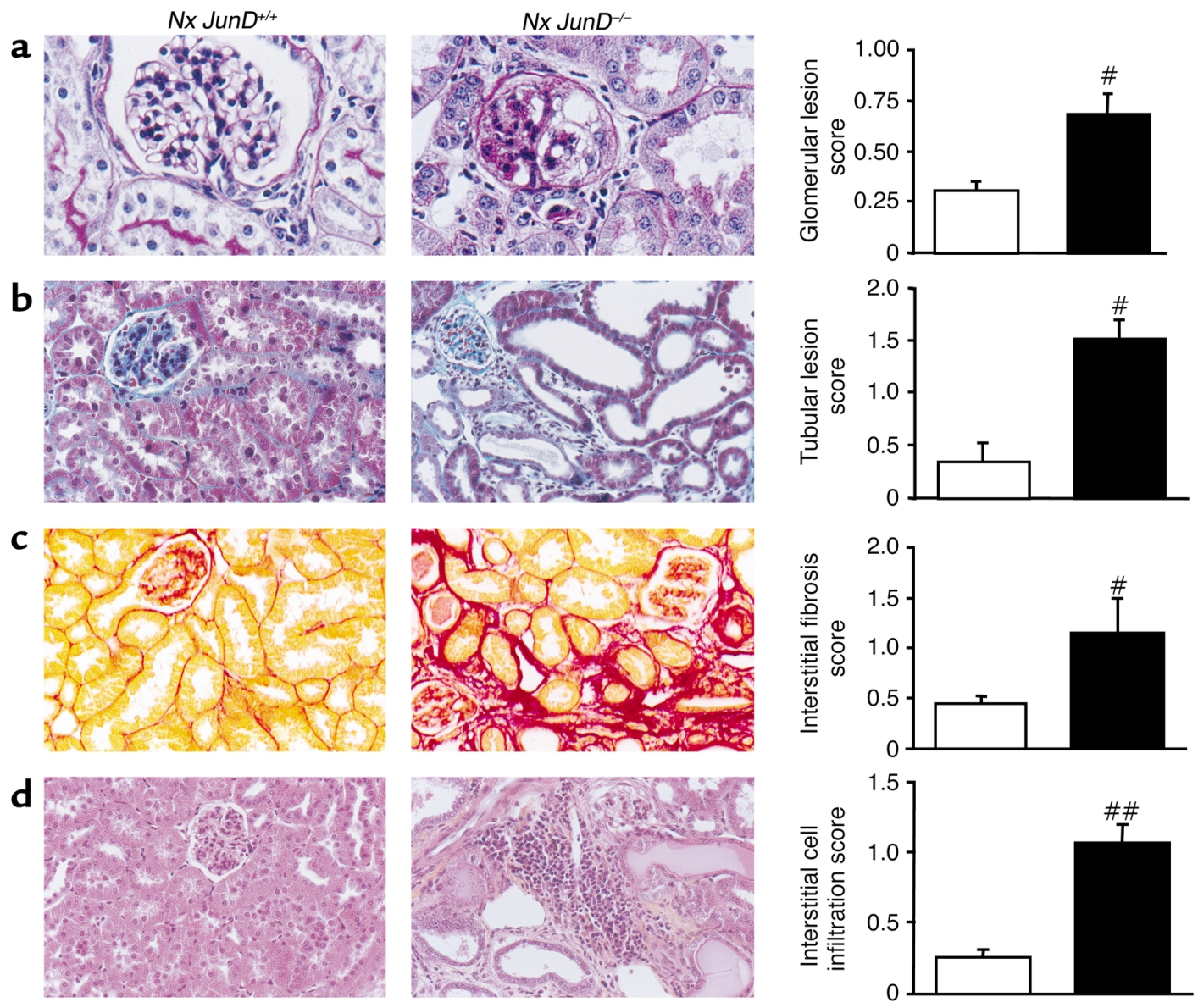

Figure 1

Accelerated renal lesion development in the absence of JunD. Morphology and lesion scores of remnant kidneys from JunD ${ }^{+/+}$(white bars) and Jun $D^{-/-}$(black bars) mice 60 days after nephron reduction $(\mathrm{Nx})$. Renal lesions were graded according to established methodology in randomly selected microscopic fields of the cortex. (a) Glomerular lesions, $\times 400$. (b) Tubular lesions, $\times 200$. (c) Interstitial fibrosis, $\times 200$. (d) Interstitial mononuclear cell infiltration, $\times 200$. Results represent mean $\pm \mathrm{SEM} ; n=6 .{ }^{\#} P<0.05,{ }^{\# \#} P<0.01, \mathrm{Jun}^{-/-}$versus JunD ${ }^{+/+}$by Mann-Whitney test.

\section{Results}

Accelerated renal lesion development in the absence of JunD. We performed $75 \%$ reduction of renal mass in $J u n D^{+/+}$ and $J u n D^{-/-}$mice and studied remnant kidneys at 7,14 , 30 , and 60 days after surgery. In the remnant kidneys of both wild-type and $J u n D^{-/}$animals we observed an early compensatory growth phase that continued up to 30 days after surgery and lead to a marked and progressive increase in remnant wet kidney weight. Compensatory growth resulted from both glomerular and tubular hypertrophy in $J u n D^{+/+}$and $J u n D^{-/-}$mice (Table 1). Hypertrophy was greater in tubules than in glomeruli, irrespective of the time point or the genotype. To perform a detailed evaluation of tubular hypertrophy, we counted the number of nuclei and calculated for each tubular section the cell number and the cell size. As shown in Table 1, tubular hypertrophy resulted from both cellular hypertrophy (increase in cell size) and cellular hyperplasia (increase in cell number) in both $J u n D^{+/+}$and $J u n D^{-/-}$mice. No differences were observed in remnant nephron hypertrophy between $\mathrm{JunD}^{+/+}$and $\mathrm{JunD}^{-/-}$mice during the first month. In wild-type animals, the compensatory growth was almost completed by day 30 . In contrast, both glomerular and tubular surfaces continued to rise dramatically at day 60 in $J u n D^{-/-}$animals. In $J u n D^{-/-}$mice only, the compensatory growth phase was followed by the appearance of severe lesions at 60 days. Lesions were mainly comprised of glomerular sclerosis and/or hyalinosis and severe tubular dilation with microcyst formation. We also observed sparse areas of interstitial fibrosis and mononuclear cell infiltration (Figure 1). Quantification of the lesions showed that $J u n D^{-/-}$mice had significantly higher scores of tubular, glomerular, and interstitial damage compared with wild-type mice (Figure 1). Taken together, these results suggest that a late phase of renal growth could favor the development of renal lesions in mutant mice.

Hypertension and proteinuria are early events after nephron reduction that precede the development of renal lesions. To evaluate whether the absence of JunD affected these parameters, we measured urinary protein excretion and blood pressure in controls and nephrectomized $J u n D^{+/+}$and $J u n D^{-/-}$mice 30 days after surgery. As expected, the mean arterial blood pressure, which was recorded in conscious animals by the tail-cuff method, 
a
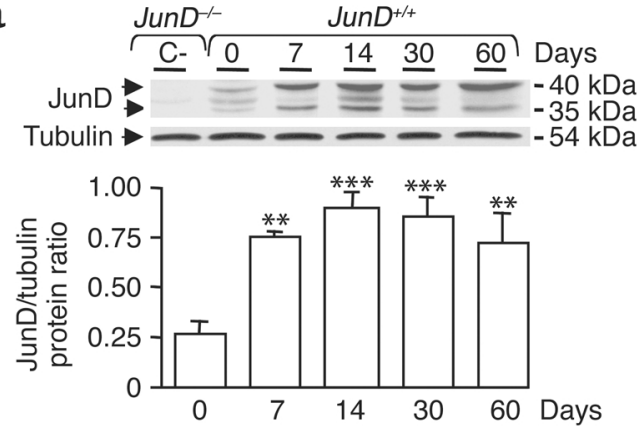

b
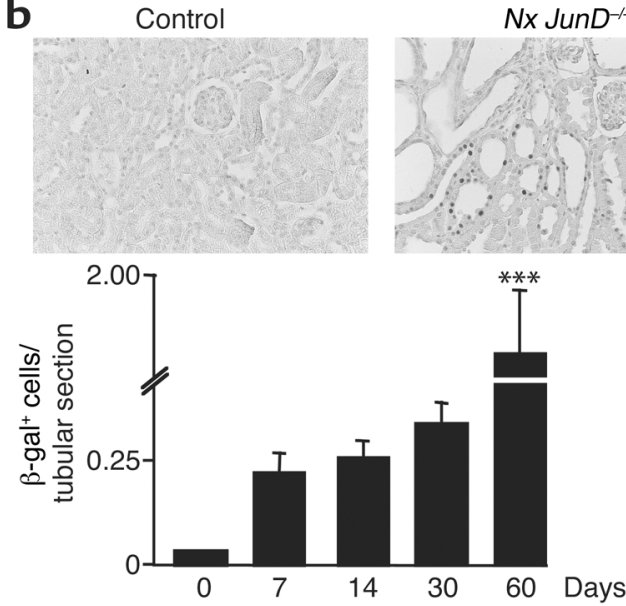

Figure 2

JunD expression increases after nephron reduction. (a) Immunoblot analysis of JunD expression in control nonoperated (designated 0 ) and remnant kidneys of $J u n D^{+/+}$mice. Protein expression was analyzed before surgery (day 0 ) and $7,14,30$, and 60 days after nephron reduction. Extracts of JunD $D^{-/-}$kidneys served as negative controls (C-). (b) Immunohistochemical analysis of Jun $D-\beta$-gal reporter gene expression in control nonoperated (designated 0 ) and remnant kidneys of Jun $D^{-/-}$mice. $\beta$-gal staining was performed before surgery (day 0$)$ and $7,14,30$, and 60 days after nephron reduction. The top panels show staining in control and remnant kidneys at day $60(\times 200)$. The bottom panel shows the number of $\beta$-gal-positive cells per tubular section at each experimental time point. Data are mean \pm SEM; $n=4-6$ for each point. ANOVA, followed by Tukey-Kramer test: ${ }^{*} P<0.005$, $* * * P<0.001$, remnant versus control kidneys.

increased after nephron reduction. The increase was of the same magnitude in $J u n D^{+/+}(147 \pm 5 \mathrm{~mm} \mathrm{Hg})$ and JunD $D^{-/}(145 \pm 6 \mathrm{~mm} \mathrm{Hg})$ mice as compared with control nonoperated animals $(121 \pm 2 \mathrm{~mm} \mathrm{Hg} ; P<0.05)$. In contrast, urinary protein excretion did not change significantly at day 30 after nephron reduction, regardless of the genotype $(3.50 \pm 0.6,6.47 \pm 0.9$, and $5.51 \pm 1.0 \mathrm{mg}$ per 24 hours in controls, nephrectomized $J u n D^{+/+}$, and nephrectomized $J u n D^{-/-}$mice, respectively).

We next analyzed JunD expression levels following nephron reduction. Western blot analysis showed that JunD levels increased significantly in remnant $J u n D^{+/+}$ kidneys compared with nonoperated controls (Figure 2). The increase was detected from day 7 after surgery and was maintained throughout the course of the experiment. In the mutant animals the junD locus has been replaced with a $\beta$-gal-Lac $Z$ cassette (22), allowing us to monitor $J u n D$ gene activation in remnant kidneys. The number of $\beta$-gal-positive nuclei increased markedly (threefold) in $J u n D^{-/-}$kidneys compared with nonoperated mice from day 7 after nephron reduction (Figure 2). The staining was maintained for the first month after surgery and increased dramatically by day 60 .

Increased tubular cell proliferation in the absence of JunD. We analyzed cell proliferation throughout the experimental time course using PCNA immunostaining as a marker of cell-cycle progression (Figure 3). In control nonoperated kidneys, we recorded a low level of tubular cell proliferation (Figure 3a). The number of PCNApositive tubular cells increased dramatically at day 7 and day 14 in remnant kidneys of both $\mathrm{JunD}^{+/+}$and Jun $D^{-/-}$mice. By day 30 , the levels had dropped back to baseline in both groups. The pattern of cell proliferation was indistinguishable in $J u n D^{+/+}$and $J u n D^{-/-}$mice during the first month after nephrectomy. By day 60 , however, there was a startling increase in cell proliferation in JunD $D^{-/-}$animals only. The number of PCNApositive cells rose dramatically (over threefold), while the levels in wild-type littermates remained unchanged. As shown in Table 1, cell proliferation resulted in increased cell number per tubular cross section in both $\mathrm{JunD}^{+/+}$and $J u n D^{-/-}$remnant kidneys from day 7 after surgery. The number of tubular nuclei did not change from day 7 to day 30 after nephrectomy. It significantly increased at 60 days, however, but exclusively in JunD $D^{-/-}$mice, confirming the second wave of cell proliferation in mutant animals. A positive correlation was found between tubular cell proliferation and the glomerular lesion, tubular lesion, interstitial fibrosis, and mononuclear cell infiltration scores in nephrectomized animals at day 60 (linear regression: $\mathrm{r}^{2}=0.453$, $0.546,0.603,0.720 ; P=0.016,0.006,0.003,0.0005$ for glomerular or tubular lesions, interstitial fibrosis, and mononuclear cell infiltration, respectively). In contrast to the marked increase in tubular cell proliferation, very few PCNA-positive cells were detected in glomeruli regardless of the genotype.

We next investigated the mechanism by which JunD regulates cell proliferation following nephron reduction. AP-1 is known to regulate cell cycle progression via regulatory proteins such as cyclin D1 (28). A radionuclease protection assay (RPA) failed to detect any changes in cyclin D1, D2, and D3 mRNAs levels, irrespective of the time point or the genotype (not shown). We then analyzed the relationship between proliferation and $j u n D$ expression at the cellular level by monitoring the coexpression of PCNA and $\beta$-gal proteins in serial sections. The number of cells expressing both PCNA and $\beta$-gal was very low in remnant kidneys of $J u n D^{-/-}$mice, regardless of the experimental time point (Figure $3 \mathrm{~b}$ ); the two proteins were either expressed by different cells of the same tubular section or in distinct tubular segments. PCNA staining was predominantly in proximal tubular cells, whereas $\beta$-gal was specifically expressed in the distal tubules. To provide additional information in favor of such localiza- 
tion, we performed colocalization experiments using appropriate markers for each tubular segment. As shown in Figure 3c, we observed that PCNA-positive nuclei were mainly located in tubules expressing the LTL, a lectin that specifically binds to proximal tubular brush border carbohydrates (29). Conversely, $\beta$-galpositive staining colocalized with Tamm-Horsfall, a well-known glycoprotein expressed in the ascending limb of Henle's loops and distal tubules (30). Thus, proliferating cells are distinct from those expressing the JunD- $\beta$-gal reporter gene, suggesting that the effect of $j u n D$ gene inactivation is not cell autonomous.

Elevated expression of TGF- $\alpha$ in the absence of JunD. Paracrine mechanisms might account for the reactivation of cell proliferation 60 days after nephron reduction in $J u n D^{-/-}$mice. The EGF pathway acts as an autocrine/ paracrine system in the kidney; distal tubular cells secrete the ligands (predominantly EGF and TGF- $\alpha$ ), whereas the receptor (EGFR) is abundantly expressed in proximal tubules $(31,32)$. We showed previously the central role of this pathway in renal deterioration $(5,9)$. Hence, we monitored the expression of EGF and TGF- $\alpha$ 60 days after nephron reduction in $J u n D^{-/-}$mice and wild-type littermates using RPA. The expression levels were comparable in nonoperated kidneys of $\mathrm{JunD}^{+/+}$and JunD $D^{-/}$mice, but changed after nephron reduction (Figure 4a). EGF mRNA levels decreased in the remnant kidneys of nephrectomized mice regardless of the genotype. In contrast, TGF- $\alpha$ mRNA increased significantly in the remnant kidneys of $J u n D^{-/-}$mice, while the levels decreased in wild-type mice.

We next performed immunohistochemistry analysis and showed cytoplasmic EGF and TGF- $\alpha$ staining in cortical distal tubules of control nonoperated $J u n D^{+/+}$and $J u n D^{-/-}$mice (Figure $4 \mathrm{~b}$ ). The EGF staining was unaffected by nephron reduction or JunD inactivation. Sixty days after nephron reduction, TGF- $\alpha$ levels increased markedly in JunD $D^{-/-}$kidneys, both in terms of the extent and the intensity of the staining. In contrast, the pattern of TGF- $\alpha$ staining did not change after nephron reduction in $\mathrm{JunD}^{+/+}$ mice. The mean staining density per tubular section was higher in $J u n D^{-/-}$kidneys $(0.532 \pm 0.008)$ than in nonoperated $(0.437 \pm 0.025)$ or wild-type remnant $(0.427 \pm 0.038)$ kidneys $(P=0.02)$. Western blot analysis detected a specific immunoreactive $37-\mathrm{kDa}$ band (absent in $T G F-\alpha^{/-}$mice) at 60 days after nephron reduction in remnant kidneys from $j u n D^{-/-}$mice, exclusively (Figure 4c). Immunohistochemistry and Western blot analysis throughout the experimental time course showed no changes in TGF- $\alpha$ staining during the first month following nephron reduction, regardless of the genotype (not shown).

Inbibition of EGFR signaling prevents lesion development and cell proliferation in JunD $D^{-/}$mice. To confirm the role of TGF- $\alpha$ in the development of renal lesions in $J u n D^{-/-}$ mice, we bred these mice with transgenic mice (REM) (9) overexpressing a dominant negative mutant isoform of EGFR selectively in renal proximal tubular cells. The inhibition of EGFR signaling dramatically reduced the development of lesions in bitransgenic JunD ${ }^{-/-} / R E M$ mice. Two months after nephron reduction, the majority of the glomeruli and tubules were normal, and very few areas of interstitial fibrosis and mononuclear cell infiltrates were observed in JunD $D^{-/} / R E M$ remnant kidneys. The score of both glomerular and tubular lesions were markedly reduced in bitransgenic mice compared with $J u n D^{-/-}$mice $(0.431 \pm 0.052$ versus $0.685 \pm 0.115$, $P<0.02$, and $0.155 \pm 0.030$ versus $1.516 \pm 0.213, P<0.001$, for glomeruli and tubules, respectively).

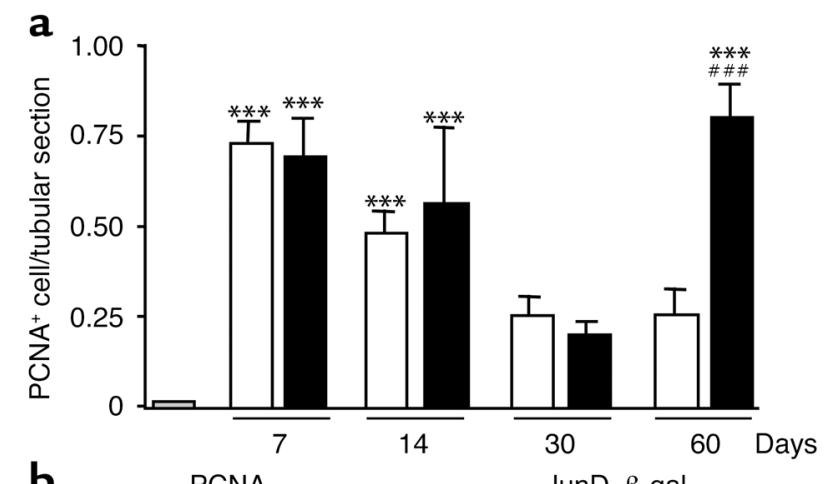

b PCNA

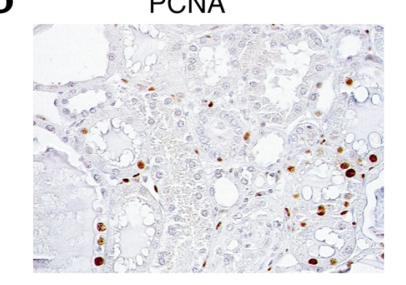

JunD- $\beta$-gal

c LTL
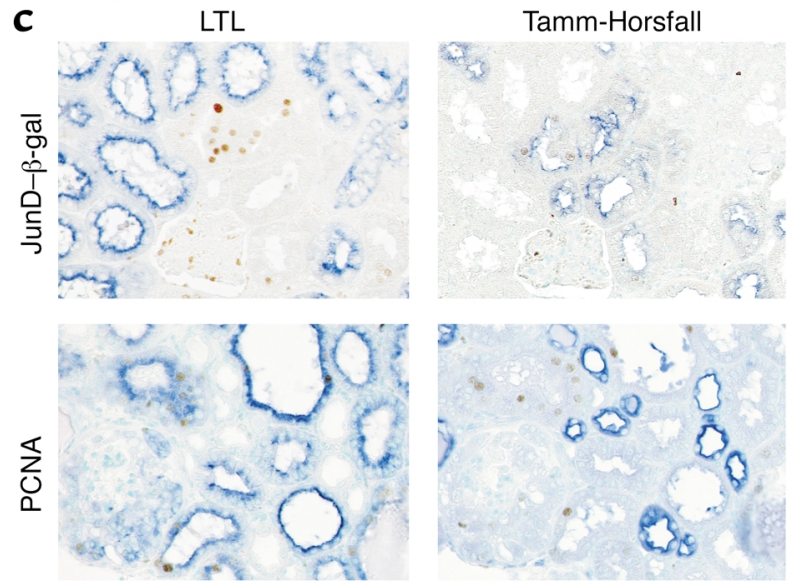

\section{Figure 3}

Increased tubular cell proliferation in the absence of JunD. (a) The number of PCNA-positive cells per tubular section was measured in control kidneys (gray bar) and in $\mathrm{JunD}^{+/+}$(white bars) and JunD ${ }^{-/-}$ (black bars) remnant kidneys 7, 14, 30, and 60 days after nephron reduction. Data are mean \pm SEM; $n=4-6$ for each time point. ${ }^{* *} P<0.001$, remnant versus control kidneys; $\# \# P<0.001$, Jun $D^{-1-}$ versus $J u n D^{+/+}$by ANOVA, followed by Tukey-Kramer test. (b) PCNA and $\beta$-gal immunostaining in serial $4-\mu \mathrm{m}$ sections of $J u n D^{-/-}$remnant kidneys at day $60(\times 200)$. (c) Colocalization experiments in serial sections of JunD ${ }^{-/-}$remnant kidneys at day 60 . Upper panels: overlay of $\beta$-gal staining and LTL staining (left) or Tamm-Horsfall staining (right). Lower panels: overlay of PCNA staining and LTL staining (left) or Tamm-Horsfall staining (right). $(\times 200)$. 


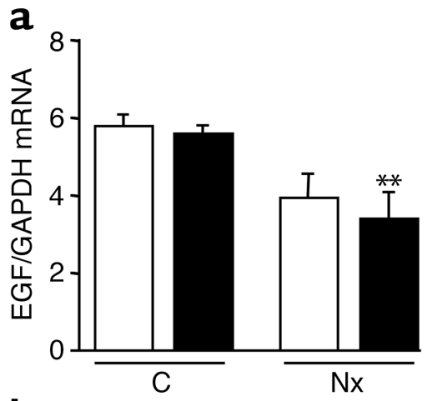

b
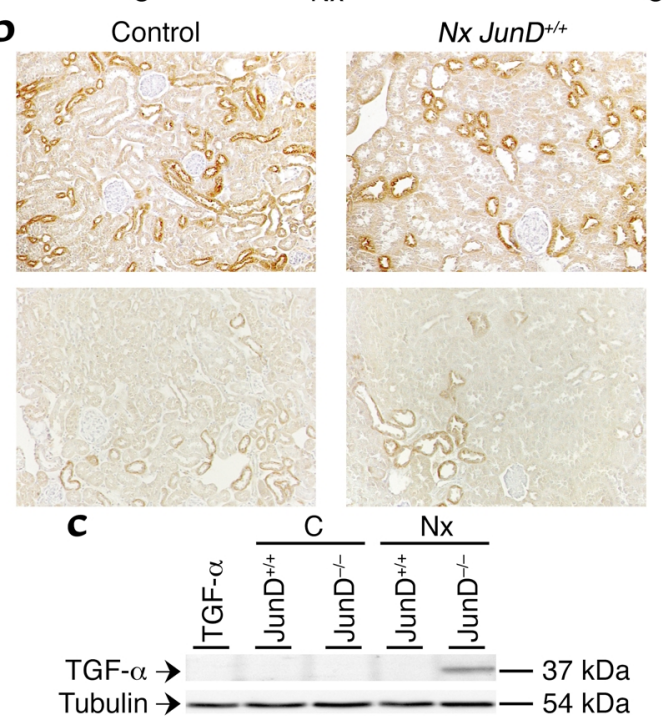
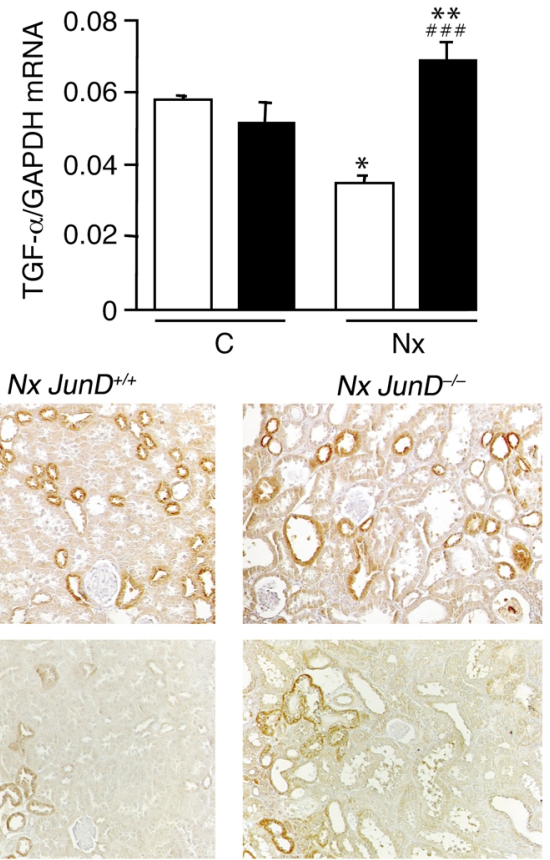

Figure 4

Elevated TGF- $\alpha$ expression in the absence of JunD. (a) EGF and TGF- $\alpha$ mRNA expression in control nonoperated $(\mathrm{C})$ and remnant $(\mathrm{Nx})$ kidneys of Jun $D^{+/+}$(white bars) and Jun $D^{-/-}$(black bars) mice at day 60 . Data are mean $\pm \mathrm{SEM} ; n=6$ for each point. ${ }^{*} P<0.05,{ }^{*} P<0.01$, remnant versus control kidneys; $\# \# P<0.001$, JunD $D^{-/-}$versus Jun $D^{+/+}$ kidneys by ANOVA, followed by Tukey-Kramer test. (b) Immunohistochemistry of EGF (upper) and TGF- $\alpha$ (lower) expression in control nonoperated and remnant $(\mathrm{Nx})$ kidneys of $J u n D^{+/+}$and Jun $D^{-/-}$mice at day $60(\times 200)$. (c) Immunoblot analysis of TGF- $\alpha$ expression in control nonoperated $(\mathrm{C})$ and remnant $(\mathrm{Nx})$ kidneys of $J u n D^{+/+}$and JunD ${ }^{-/-}$mice at day 60 . Extracts of TGF- $\alpha^{-1-}$ kidneys served as negative controls.
Immunostaining (Figure 5b) showed that the number of tubular PCNA-positive cells was significantly lower in bitransgenic $J u n D^{-/-} / R E M$ mice than in $j u n D^{-/-}$ mice $(0.126 \pm 0.009$ versus $0.806 \pm 0.090, P<0.001)$ at 60 days and did not differ significantly from nonoperated controls.

\section{Discussion}

Genetically modified mice offer a powerful approach to define specific roles for individual factors in the complex pathogenesis of renal deterioration. We combined a genetic approach (JunD $D^{-/-}$mice) and an experimental model of renal injury $(75 \%$ reduction of total renal mass) to reveal a key role for JunD/AP-1 in regulating the development of renal lesions. JunD is induced by nephron reduction and is linked to inhibition of a second wave of cell proliferation at the moment of parenchyma deterioration. At this stage, JunD prevents the overexpression of TGF- $\alpha$, a potent mitogen of renal cells. Expression of a dominant negative EGFR isoform prevented the hyperproliferation and lesion development in JunD $D^{-/-}$mice. Thus, JunD acts as an essential switch to turn off cell proliferation after nephron reduction once compensatory growth is achieved, possibly through the inhibition of paracrine factors involved in the cross-talk between renal cells.

The marked increase in cell proliferation 2 months after nephron reduction may account for the development of lesions in $J u n D^{-/-}$mutant mice. This is supported by the finding that selective inhibition of proliferation in prox-

\section{Figure 5}

Overexpression of a dominant negative EGFR prevents lesion development and cell proliferation in JunD $D^{-/-}$ mice. (a) Morphology of control and of remnant $(\mathrm{Nx})$ kidneys from $J u n D^{-/-}$and $J u n D^{-/-} / R E M$ mice at day 60 . PAS staining, $\times 200$. (b) Immunohistochemical analysis of PCNA in control and in remnant $(\mathrm{Nx})$ kidneys from JunD $D^{-/-}$and JunD ${ }^{-/-} /$REM mice at day $60(\times 200)$.
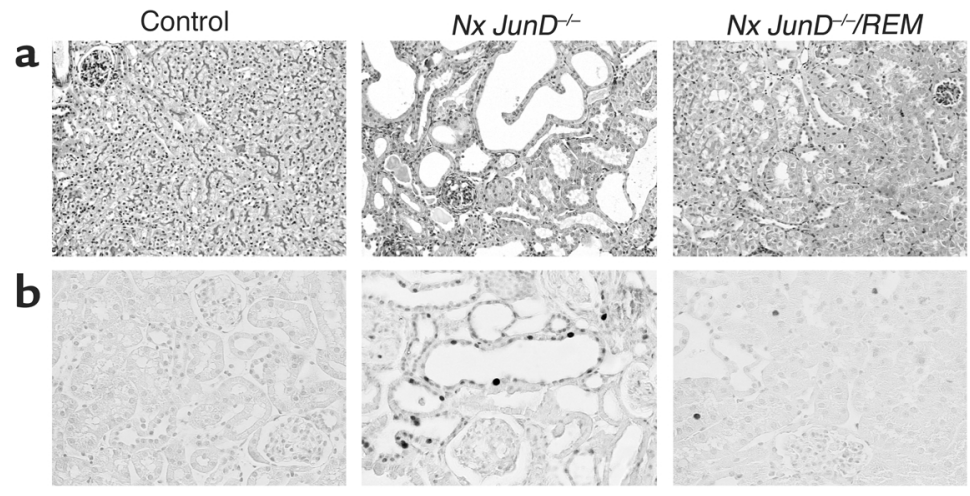
imal tubules prevents the development of renal lesions in bitransgenic JunD $D^{-/} / R E M$ mice. Moreover, several experimental and clinical observations suggest that cell proliferation plays a key role in the destruction of kidneys during chronic disease. First, proliferation of mesangial, tubular, and interstitial cells increases in many renal diseases and correlates with the severity of renal lesions (2-5). Secondly, we have shown that the development of renal lesions parallels the extent of cell proliferation in two mouse strains (FVB/N and C57Bl6 $\times \mathrm{DBA} 2 / \mathrm{F} 1)$ and in two experimental models (unilateral and subtotal nephrectomy) that respond differently to nephron loss $(3,26)$. Thirdly, treatments that delay the progression of renal lesions (such as pharmacological inhibitors or diet) reduce the rate of cell proliferation in injured kidneys (5, $7,24)$. It is noteworthy that the absence of JunD affects neither the development of hypertension nor the appearance of proteinuria, two factors that have been proposed to account for the development of renal lesions after nephron reduction.

Studies of fibroblasts in culture have indicated that JunD can act as a negative regulator of cell proliferation $(13,23)$. Here we provide the first evidence for a similar role in vivo and demonstrate that JunD prevents renal cell proliferation indirectly by inhibiting the expression of paracrine factors. Indeed, we show that cells expressing the JunD- $\beta$-gal reporter gene are distinct from those expressing PCNA. PCNA-positive cells were predominantly in proximal tubules, the nephron segment that undergoes dilation during the deterioration process, whereas $\beta$-gal staining was detected in distal tubules, the site of growth factor production. This supports earlier reports that distal tubular cells overexpressing Jun/Fos proteins are distinct from proximal tubular cells undergoing DNA synthesis in the postischemic kidney (33). Moreover, we show that the expression of cyclin D1 mRNA encoding a key regulator of the G1/S transition was not affected in $J u n D^{-/-}$kidneys. It is possible that other cell cycle regulators are involved in the kidney proliferation under pathological conditions (34). Finally, we observed that (i) TGF- $\alpha$ expression increased in distal tubules after nephron reduction in $J u n D^{-1-}$ mice and that (ii) functional inactivation of the TGF- $\alpha$ receptor in proximal tubules, by a dominant negative transgenic strategy, prevents the increase in cell proliferation in bitransgenic $J u n D^{-1-} / R E M$ mice. The increase in TGF- $\alpha$ is specific since the expression of EGF, another distal tubule mitogen that binds the same receptor, is not affected by JunD inactivation. Interestingly, the increase in TGF- $\alpha$ protein levels is higher than that of the transcript, suggesting that, in our experimental conditions, TGF- $\alpha$ expression may be regulated by both translation and transcriptional control. Previous in vitro studies showed that regulation of TGF- $\alpha$ expression is a complex phenomenon that involves several steps, including the control of mRNA transcription, mRNA stability, protein translation, and pro-TGF- $\alpha$ processing (35). Each of these events may become the predominant checkpoint, according to the type of cell and/or to the pathological condition. The mechanism by which JunD regulates TGF- $\alpha$ expression is unclear and merits further investigation.

Activation of the EGFR pathway has been implicated in the evolution of renal disease. Overexpression of an active EGFR form, the c-erb-B2 receptor, induces tubular hyperplasia and the development of renal cysts in transgenic mice (36). Conversely, expression of the dominant negative EGFR isoform in proximal tubules inhibits tubular cell proliferation and interstitial collagen accumulation leading to reduced glomerular and tubulo-interstitial lesions after nephron reduction (9). Other genetic approaches (crossing mouse strains with orpk cystic and waved-2 EGFR mutations) have confirmed the role of EGFR in tubular cyst formation and cell proliferation (37). Furthermore, both EGF and TGF- $\alpha$, the two principal EGFR ligands in the kidney, increase in several renal diseases, but the contribution of each factor has not been investigated (38). Our results suggest that TGF- $\alpha$, rather than EGF, is involved in destruction of the renal parenchyma. This is supported by transgenic mice studies in which overexpression of TGF- $\alpha$ induced severe renal lesions (39), whereas transgenic mice broadly overexpressing EGF do not develop a renal phenotype (40).

During the first month following nephrectomy, the initial compensatory renal growth, as judged by kidney weight, glomerular and tubular hypertrophy, PCNA staining, and nuclei counting, was not affected by the absence of JunD. It should be pointed out that the early compensatory response phase was similar in terms of intensity, nature (increase in cell size and number), and compartments (glomerular and tubular overgrowth) in $J u n D^{+/+}$and $J u n D^{-/-}$remnant kidneys. The observation that, in contrast to the early phase, only the number of cells per tubule increased at day 60 in mutant mice, whereas the cell size did not change, suggests a predominant role of tubular hyperplasia rather than hypertrophy in lesion development. It also appears that distinct molecular events might regulate the initial compensatory growth phase and the late proliferation associated with the development of renal lesions. It is noteworthy that, in several lesion-resistant mouse strains, including those used in the present study, neither increased cell proliferation nor lesion development could be detected until at least 6 months after nephron reduction (unpublished data). The present study provides the first evidence of a second wave of cell proliferation in the absence of JunD. We propose that the inhibition of cell proliferation following the compensatory phase is critical to prevent pathological lesions. JunD represents the first example of a gene functioning at this critical turning point. The factor(s) that could be involved directly or indirectly in the delayed effect of $J u n D$ gene inactivation remain(s) to be elucidated.

Studies in different mouse strains have highlighted a genetic component to the development of renal lesions. Previous data showed that nephron reduction induces pathological lesions in $\mathrm{FVB} / \mathrm{N}$ or ragged 
oligosyndactyly pintail (ROP) mice, whereas other strains (e.g., C57Bl6 and C57B16 $\times$ DBA2/F1) are resistant to early renal deterioration $(26,41,42)$. Mild lesions were apparent, however, in some resistant strains 8 months after nephron reduction (9). Genetic background affects the severity of renal phenotypes in other experimental models of renal diseases, such as polycystic kidney disease or Alport nephropathy (43, 44 ). The mice used in our study have a mixed $129 / \mathrm{Sv} x$ C57Bl6 background from two strains that are resistant to early renal deterioration. Megyesi et al. reported that $129 / \mathrm{Sv}$ mice develop renal lesions after nephron reduction, but tubulo-interstitial lesions were mild and only apparent 14-16 weeks after surgery, much later than the present protocol (45). It is possible that AP-1 components are involved, directly or indirectly, in susceptibility to renal disease; that is, JunD loss can transform resistant mice into lesionprone animals. Although we are aware that the mixed genetic background of the mice might affect the development of renal lesions, the consistent use of matched littermates and the complete rescue by EGFR transgenic mice suggest that genetic background does not account for the dramatic phenotype. It is noteworthy that the evolution of renal disease varies widely in patients, from very slow to fast progressors (46), but the genetic factors remain unknown.

In conclusion, previous in vitro studies support a role for JunD as a negative regulator of proliferation and cell cycle progression. Here, we provide what we believe to be the first evidence for a similar role in vivo and demonstrate that JunD-mediated inhibition of proliferation may prevent hyperplasia associated with pathological conditions. Furthermore, our study has highlighted a novel paracrine mechanism for AP-1 regulation of cell proliferation, expanding the complex network of AP-1 cellular control. Finally, we have identified an experimental model that may help us (i) to identify signaling components linked to susceptibility to renal disease with a different clinical time course in human patients and (ii) to design therapeutic strategies to treat the growing number of patients with chronic renal failure.

\section{Acknowledgments}

We are grateful to Stephanie Lecorre, Martine MuffatJoly, and Agnes Legrand for technical assistance at Centre d'Explorations Fonctionelles Intégrées, Institut Fédératif de Recherche 2. We thank D.C. Lee for $\mathrm{TGF}^{-1}$ kidneys, B. Lardeux for RPA probes, P. Briand for critical advice, and P. Verpillat for statistical advice. This work was supported by Institut National de la Santé et de la Recherche Médicale, Université René Descartes, Laboratoires de Recherches Physiologiques, Association pour la Recherche contre le Cancer (grant 9896), Centre de Recherche Industrielle et Technique, European Community Training \& Mobility of Researchers Program, and the Association for International Cancer Research.
1. Hostetter, T.H. 1995. Progression of renal disease and renal hypertrophy. Annu. Rev. Physiol. 57:263-278.

2. Olivetti, G., Anversa, P., Rigamonti, W., Vitali-Mazza, L., and Loud, A.V. 1977. Morphometry of the renal corpuscle during normal postnatal growth and compensatory hypertrophy. A light microscope study. J. Cell Biol. 75:573-585.

3. Terzi, F., et al. 1995. Subtotal but not unilateral nephrectomy induces hyperplasia and protooncogene expression. Am. J. Physiol. 268:F793-F801.

4. Kliem, V., et al. 1996. Mechanisms involved in the pathogenesis of tubulointerstitial fibrosis in 5/6-nephrectomized rats. Kidney Int. 49:666-678.

5. Terzi, F., et al. 2000. Sodium restriction decreases AP-1 activation after nephron reduction in the rat: role in the progression of renal lesions. Exp. Nephrol. 8:104-114.

6. Ishidoya, S., Morrissey, J., McCracken, R., Reyes, A., and Klahr, S. 1995. Angiotensin II receptor antagonist ameliorates renal tubulointerstitial fibrosis caused by unilateral ureteral obstruction. Kidney Int. 47:1285-1294.

7. Johnson, R.J., et al. 1992. Inhibition of mesangial cell proliferation and matrix expansion in glomerulonephritis in the rat by antibody to platelet-derived growth factor. J. Exp. Med. 175:1413-1416.

8. Kashihara, N., Maeshima, Y., and Makino, H. 1997. Therapeutic intervention in glomerulonephritis by oligonucleotides. Exp. Nephrol. 5:126-131.

9. Terzi, F., et al. 2000. Targeted expression of a dominant-negative epidermal growth factor receptor in the kidney reduces tubulo-interstitial lesions after renal injury. J. Clin. Invest. 106:225-234.

10. Angel, P., and Karin, M. 1991. The role of Jun, Fos and the AP-1 complex in cell-proliferation and transformation. Biochim. Biophys. Acta. 1072:129-157

11. Hirai, S.I., Ryseck, R.P., Mechta, F., Bravo, R., and Yaniv, M. 1989. Characterization of junD: a new member of the jun proto-oncogene family. EMBO J. 8:1433-1439.

12. Lallemand, D., Spyrou, G., Yaniv, M., and Pfarr, C.M. 1997. Variations in Jun and Fos protein expression and AP-1 activity in cycling, resting and stimulated fibroblasts. Oncogene. 14:819-830.

13. Pfarr, C.M., et al. 1994. Mouse JunD negatively regulates fibroblast growth and antagonizes transformation by ras. Cell. 76:747-760.

14. Safirstein, R., Price, P.M., Saggi, S.J., and Harris, R.C. 1990. Changes in gene expression after temporary renal ischemia. Kidney Int. 37:1515-1521.

15. Ingram, A.J., and Scholey, J.W. 1997. Protooncogene expression and diabetic kidney injury. Kidney Int. Suppl. 60:S70-S76.

16. Hamaguchi, A., Kim, S., Izumi, Y., and Iwao, H. 2000. Chronic activation of glomerular mitogen-activated protein kinases in Dahl salt-sensitive rats. J. Am. Soc. Nephrol. 11:39-46.

17. Cowley, B.D., Jr., Chadwick, L.J., Grantham, J.J., and Calvet, J.P. 1991. Elevated proto-oncogene expression in polycystic kidneys of the C57BL/6J (cpk) mouse. J. Am. Soc. Nephrol. 1:1048-1053.

18. Sakurai, H., et al. 1997. Suppression of NF-kappa B and AP-1 activation by glucocorticoids in experimental glomerulonephritis in rats: molecular mechanisms of anti-nephritic action. Biochim. Biophys. Acta. 1362:252-262.

19. Muller, D.N., et al. 2000. Effect of bosentan on NF-kappaB, inflammation, and tissue factor in angiotensin II-induced end-organ damage. Hypertension. 36:282-290.

20. Park, J.K., et al. 2000. Cerivastatin prevents angiotensin II-induced renal injury independent of blood pressure- and cholesterol-lowering effects. Kidney Int. 58:1420-1430.

21. Jochum, W., Passegue, E., and Wagner, E.F. 2001. AP-1 in mouse development and tumorigenesis. Oncogene. 20:2401-2412.

22. Thepot, D., et al. 2000. Targeted disruption of the murine junD gene results in multiple defects in male reproductive function. Development. 127:143-153.

23. Weitzman, J.B., Fiette, L., Matsuo, K., and Yaniv, M. 2000. JunD protects cells from p53-dependent senescence and apoptosis. Mol. Cell. 6:1109-1119.

24. Terzi, F., Beaufils, H., Laouari, D., Burtin, M., and Kleinknecht, C. 1992. Renal effect of anti-hypertensive drugs depends on sodium diet in the excision remnant kidney model. Kidney Int. 42:354-363.

25. Raij, L., Azar, S., and Keane, W. 1984. Mesangial immune injury, hypertension, and progressive glomerular damage in Dahl rats. Kidney Int. 26:137-143.

26. Pillebout, E., et al. 2001. Proliferation and remodeling of the peritubular microcirculation after nephron reduction: association with the progression of renal lesions. Am. J. Pathol. 159:547-560.

27. Kaabache, T., Barraud, B., Feldmann, G., Bernuau, D., and Lardeux, B. 1995. Direct solution hybridization of guanidine thiocyanate-solubilized cells for quantitation of mRNAs in hepatocytes. Anal. Biochem. 232:225-230.

28. Herber, B., Truss, M., Beato, M., and Muller, R. 1994. Inducible regulatory elements in the human cyclin D1 promoter. Oncogene. 9:2105-2107.

29. Laitinen, L., Virtanen, I., and Saxen, L. 1987. Changes in the glycosylation pattern during embryonic development of mouse kidney as revealed 
with lectin conjugates. J. Histochem. Cytochem. 35:55-65

30. Ivanyi, B., and Olsen, T.S. 1991. Immunohistochemical identification of tubular segments in percutaneous renal biopsies. Histochemistry. 95:351-356

31. Nouwen, E.J., and De Broe, M.E. 1994. EGF and TGF-alpha in the human kidney: identification of octopal cells in the collecting duct. Kidney Int. 45:1510-1521.

32. Breyer, M.D., Redha, R., and Breyer, J.A. 1990. Segmental distribution of epidermal growth factor binding sites in rabbit nephron. Am. J. Physiol. 259:F553-F558.

33. Megyesi, J., Di Mari, J., Udvarhelyi, N., Price, P.M., and Safirstein, R. 1995. DNA synthesis is dissociated from the immediate-early gene response in the post-ischemic kidney. Kidney Int. 48:1451-1458.

34. Shankland, S.J., Hamel, P., and Scholey, J.W. 1997. Cyclin and cyclindependent kinase expression in the remnant glomerulus. J. Am. Soc. Nephrol. 8:368-375.

35. Lee, D.C., Fenton, S.E., Berkowitz, E.A., and Hissong, M.A. 1995. Transforming growth factor alpha: expression, regulation, and biological activities. Pharmacol. Rev. 47:51-85.

36. Stocklin, E., Botteri, F., and Groner, B. 1993. An activated allele of the c-erbB-2 oncogene impairs kidney and lung function and causes early death of transgenic mice. J. Cell Biol. 122:199-208.

37. Richards, W.G., et al. 1998. Epidermal growth factor receptor activity mediates renal cyst formation in polycystic kidney disease. J. Clin. Invest. 101:935-939.
38. Terzi, F., Burtin, M., and Friedlander, G. 1998. Early molecular mechanisms in the progression of renal failure: role of growth factors and protooncogenes. Kidney Int. Suppl. 53:S68-S73.

39. Lowden, D.A., et al. 1994. Renal cysts in transgenic mice expressing transforming growth factor-alpha. J. Lab. Clin. Med. 124:386-394.

40. Wong, R.W., et al. 2000. Overexpression of epidermal growth factor induced hypospermatogenesis in transgenic mice. J. Biol. Chem. 275:18297-18301.

41. Esposito, C., He, C.J., Striker, G.E., Zalups, R.K., and Striker, L.J. 1999 Nature and severity of the glomerular response to nephron reduction is strain-dependent in mice. Am. J. Pathol. 154:891-897.

42. Kren, S., and Hostetter, T.H. 1999. The course of the remnant kidney model in mice. Kidney Int. 56:333-337.

43. Iakoubova, O., Dushkin, H., Pacella, L., and Beier, D.R. 1999. Genetic analysis of modifying loci on mouse chromosome 1 that affect disease severity in a model of recessive PKD. Physiol. Genomics. 1:101-105.

44. Andrews, K.L., Mudd, J.L., Li, C., and Miner, J.H. 2002. Quantitative trait loci influence renal disease progression in a mouse model of Alport syndrome. Am. J. Pathol. 160:721-730.

45. Megyesi, J., Price, P.M., Tamayo, E., and Safirstein, R.L. 1999. The lack of a functional p21(WAF1/CIP1) gene ameliorates progression to chronic renal failure. Proc. Natl. Acad. Sci. U. S. A. 96:10830-10835.

46. Schelling, J.R., Zarif, L., Sehgal, A., Iyengar, S., and Sedor, J.R. 1999. Genetic susceptibility to end-stage renal disease. Curr. Opin. Nephrol. Hypertens. 8:465-472. 